DOI: https://doi.org/10.46296/yc.v5i9ucedespsoct.0124

\title{
CARACTERIZACIÓN DE LA FERMENTACIÓN ALCOHÓLICA DEL LACTOSUERO A ESCALA DE LABORATORIO
}

\section{CHARACTERIZATION OF THE ALCOHOLIC FERMENTATION OF WHEY ON A LABORATORY SCALE}

\author{
Cardona-Soberao Yolexis Roberta 1; Cruz-Carbonell Mérida Esmunda 2; \\ González-Zambrano José Miguel ${ }^{3}$; Paneque-Díaz Yurisdan ${ }^{4}$
}

\begin{abstract}
${ }^{1}$ Profesor Titular del Departamento Ciencia y Tecnología de los Alimentos de la Universidad de Camagüey "Ignacio Agramonte Loynaz". Camagüey, Cuba. Correo: yolexis.cardona@reduc.edu.cu. ORCID ID: https://orcid.org/0000-0002-0042-5805
\end{abstract}

${ }^{2}$ Profesor Asistente del Departamento Ciencia y Tecnología de los Alimentos de la Universidad de Camagüey "Ignacio Agramonte Loynaz". Camagüey, Cuba. Correo: merida.cruz@reduc.edu.cu. ORCID ID: https://orcid.org/0000-0001-9283-1108

${ }^{3}$ Ingeniero Agrícola. Tecnólogo Agrícola. Ecuador. Correo:

miguellgonzalezz9@gmail.com. ORCID ID: https://orcid.org/0000-0002-9567-3309

${ }^{4}$ Estudiante de quinto año de Ingeniería Química de la facultad de Ciencias Aplicadas, Universidad de Camagüey "Ignacio Agramonte Loynaz". Camagüey, Cuba.

\section{Resumen}

Este trabajo tiene como objetivo caracterizar la fermentación alcohólica del lactosuero a escala de laboratorio. Se llevó a cabo en la Planta Piloto de la Universidad de Camagüey. Mediante un balance de masa se calcularon las cantidades de lactosa necesarias para estandarizar el suero hasta concentraciones de 50, 75, 100 y $125 \mathrm{~g} / \mathrm{l}$ de lactosa. La levadura del género Saccharomyces cerevisiae utilizado por la Industria Alcoholera Cubana no fermenta la lactosa como sustrato, por lo que se hizo necesario hidrolizarla siguiendo el procedimiento de hidrólisis ácida, para comprobar el resultado de la hidrólisis se realizaron pruebas de Polarimetría, Colorimetría y Cromatografía. Las fermentaciones se realizaron con dos réplicas a cada concentración estudiada, a las cuales se les añadió fosfato de amonio y levadura. Los resultados de hidrólisis parcial de la lactosa permitieron obtener en el proceso fermentativo porcientos en volúmenes de etanol por encima de los propuestos a partir de concentraciones de lactosa de 75 $\mathrm{g} / \mathrm{l}$, el incremento de las concentraciones de lactosa hasta $125 \mathrm{~g} / \mathrm{l}$ estuvo en correspondencia con los mejores rendimientos y productividad del proceso fermentativo. La duración de la fermentación mantuvo un crecimiento de los microorganismos con la transformación del sustrato y generación del CO2 según lo previsto y registrado en la literatura.

Palabras claves: fermentación alcohólica; lactosuero; hidrólisis.

\begin{abstract}
This work aims to characterize the alcoholic fermentation of whey on a laboratory scale. It was carried out in the Pilot Plant of the University of Camagüey. By means of a mass balance, the amounts of lactose necessary to standardize the serum were calculated to concentrations of 50 , 75,100 and $125 \mathrm{~g} / \mathrm{I}$ of lactose. The yeast of the genus Saccharomyces cerevisiae used by the Cuban Alcohol Industry does not ferment lactose as a substrate, so it was necessary to hydrolyze it following the acid hydrolysis procedure, to check the result of the hydrolysis Polarimetry, Colorimetry and Chromatography tests were carried out. Fermentations were carried out with two replicates at each concentration studied, to which ammonium phosphate and yeast were added. The results of partial hydrolysis of lactose allowed to obtain in the fermentation process
\end{abstract}

Información del manuscrito:

Fecha de recepción: 26 de julio de 2021.

Fecha de aceptación: 29 de septiembre de 2021.

Fecha de publicación: 11 de octubre de 2021. 
percentages in volumes of ethanol above those proposed from lactose concentrations of $75 \mathrm{~g} / \mathrm{l}$, the increase in lactose concentrations up to $125 \mathrm{~g} / \mathrm{I}$ was in correspondence with the best yields and productivity of the fermentation process. The duration of the fermentation maintained a growth of the microorganisms with the transformation of the substrate and the generation of $\mathrm{CO} 2$ as foreseen and registered in the literature.

Keywords: alcoholic fermentation; whey; hydrolysis.

\section{Introducción}

En el proceso productivo de los alimentos, además del producto deseado, se generan subproductos y residuos que, utilizados adecuadamente representan un medio eficaz para la obtención de beneficios económicos y ambientales por parte de las empresas productoras en particular y de la sociedad en general.

Uno de los subproductos más generados a nivel mundial producto de la transformación agroindustrial, es el lactosuero, generándose aproximadamente un $90 \%$ de masa total de la leche utilizada. El mismo retiene cerca de $55 \%$ del total de sólidos totales de la leche (Rodríguez y Aldo, 2017).

Parra (2009) define al lactosuero como un subproducto líquido obtenido después de la precipitación de la caseína durante la elaboración del queso; conteniendo principalmente lactosa, proteínas, minerales, vitaminas y grasa. Panesar et al. (2010) aportan su definición como un subproducto del queso y que éste representa alrededor del 85 a $90 \%$ del volumen de la leche procesada y retiene cerca del $50 \%$ del total de los nutrientes que contiene la leche, entre estos nutrientes se pueden mencionar lactosa, grasas, sales minerales y proteínas solubles.

El lactosuero es una fuente importante de contaminación ambientalpor su alto contenido de materia orgánica. El vertimiento del lactosuero en fuentes hídricas ocasiona que el agua se quede sin oxígeno, por causa de la acción microbiana que transforma la materia orgánica en compuestos que reducen el $\mathrm{pH}$ del agua causando como consecuencia la producción de malos olores y muerte de organismos acuáticos. (Londoño et al.2008). 
El problema al que se enfrenta la sociedad hoy en día frente al desperdicio de estos residuos agroindustriales es que no existe un claro aprovechamiento e industrializado debido al desconocimiento por parte de grandes, medianos y pequeños productores que son los que generan estos residuos, producto de la actividad agroindustrial, además que falta capacidad tecnológica y recursos económicos para darles un destino final. (Espinoza y Mendieta, 2018).

En Cuba se obtienen anualmente más de 100 millones de litros de suero de queso y a pesar de los constantes esfuerzos aún no se ha logrado un aprovechamiento racional de los valiosos componentes de este producto. Estos grandes volúmenes de lactosuero que se generan, por su alto valor nutritivo, pudiera ser utilizado para el consumo humano y se usa poco con este fin, por lo que su aprovechamiento se torna pobre en este sentido. El suero lácteo constituye una materia prima barata y de fácil obtención, pero la escasa información del mismo como alternativa de ser utilizado para la obtención de otros productos, como es el caso de la producción de etanol, lo vuelve cada vez más inseguro en cuanto a su utilidad.

La fermentación del lactosuero enfocada a la producción de etanol, puede presentarse como alternativa viable de aprovechamiento de este residuo para la generación de un producto con mayor valor agregado. (Vargas 2017).

El lactosuero es un subproducto del cual se puede obtener valor agregado al crear bebidas alcohólicas o bebidas saborizadas. Asimismo, existen varios factores que determinan la capacidad de llevar a cabo este proceso y es importante destacar como lo menciona Lawton y Alcaine (2019), que a la lactosa se le puede usar como un producto adjunto, como fuente de azúcar fermentable en la producción de etanol.

Aráuz, M. (2020) citando a Trevan (1990) expone que la producción de etanol se puede realizar a través de rutas químicas (hidratación catalítica del etileno) y por la ruta biotecnológica (fermentación). Mediante la fermentación se utiliza a microorganismos capaces de 
producir etanol por medio desustratos disponibles, tales como lactosa, xilosa y almidón. A través de procesos biotecnológicos se busca la producción de etanol por medio del suero de leche, el cual es generado como residuo de la preparación de un producto lácteo.

La obtención de etanol a partir de lactosuero es considerada un recurso energético sostenible, con alta viabilidad técnica, que brinda ventajas medioambientales y económicas a largo plazo, obtenido de la fermentación realizada por los microorganismos los cuales metabolizan los azúcares provenientes de subproductos de grandes procesos industriales (Jácquez et al. 2015), citado por Aráuz, M. (2020). Es de suma importancia recalcar el valor que tiene el lactosuero, ya que como sustrato fermentable generaría una oportunidad de desarrollo para nuevas formas de producción y cuidado con el medio ambiente.

La presente investigación tiene como finalidad aprovechar el lactosuero, para la obtención de etanol, como alternativa para el uso de este producto; teniendo en cuenta que será procesado utilizando técnicas y métodos apropiados para la obtención de un producto de calidad. Con esto, se procura aportar mayores ingresos en la utilización subproductos de la actividad agroindustrial y por tanto a disminuir la contaminación ambiental. Además, con esta alternativa de producción se dinamizará la actividad económica con la generación de fuentes de empleo y los beneficios sociales que aporta.

En Cuba es escasa la utilización del suero lácteo como materia prima para obtener otros productos, por la poca información que se tiene acerca del tema y es pobre la empleo de este para la obtención de etanol. Pero cabe destacar que, con la situación económica existente y los usos tan diversos del etanol en tantos renglones, la obtención de alcohol a partir de suero, que es una materia prima barata que se está desechando, sería un método que puede utilizarse con los grandes beneficos ya mencionados.

Por lo antes expuesto se realiza este trabajo con el objetivo: Caracterizar la fermentación alcohólica del lactosuero a escala de laboratorio. 


\section{Metodología}

La investigación fue llevada a cabo en la Planta Piloto de la Universidad de Camagüey en el período comprendido entre marzo y julio del 2020. Para la realización de la misma se hizo un convenio con la Empresa de Productos Lácteos de la Provincia Camagüey y la Empresa de Bebidas y Licores las cuales facilitaron las materias primas necesarias e imprescindibles para la realización de los experimentos.

Las materias primas utilizadas fueron: suero de leche procedente de la fábrica "La Vaquita" la cual se transportó a temperatura ambiente y en un corto período de tiempo para evitar la contaminación o proliferación de microorganismos, se utilizó también la levadura Saccharomyces cerevisiae cervecera que se transportó y conservó a una temperatura de $6^{\circ} \mathrm{C}$ y levadura Saccharomyces cerevisiae sólida que se utiliza para la producción de vinos. Se empleó lactosa en polvo para estandarizar el suero a diferentes concentraciones de lactosa, además de Fosfato de amonio (NH3PO4) como nutriente para la fermentación. Se utilizaron además soluciones de Ácido
Clorhídrico $(\mathrm{HCl})$ y de Hidróxido de Sodio $(\mathrm{NaOH})$ para hidrolizar la lactosa y neutralizar el medio.

Para caracterizar el suero se utilizó el equipo Ilamado Lacto Star marca GERBER, que emplea un procedimiento termo-óptico para determinar los componentes, midiendo de esta manera tanto las propiedades ópticas como las térmicas de las muestras y se obtuvieron los componentes de grasa y sólidos no grasos. El contenido de grasa específicamente se obtuvo con la medición de la turbidez de las muestras en conjunto con el contenido de proteínas, lo cual no es más que los componentes que no fueron disueltos en la muestra, y el contenido en proteínas se determinó particularmente mediante la diferencia entre el resultado de la medición óptica y el contenido de grasa en la medición térmica. Se pudo pronosticar además el punto de congelación de las muestras.

Para realizar la medición se colocó desde abajo un recipiente de muestra en el portarrecipientes, de manera que el tubo aspirador se introdujo en la muestra de suero hasta alcanzar por lo menos $30 \mathrm{~mm}$ de profundidad. Una vez realizado el 
proceso de aspiración se retiró el recipiente. El volumen de la muestra es de $20 \mathrm{ml}$ y solo se aspiraron $10 \mathrm{ml}$ de la muestra para la medición.

Se realizaron dos mediciones a cada muestra para obtener valores más exactos y seguros, a partir de los cuales se obtuvo especialmente el contenido de lactosa, los cuales fueron promediados mediante cálculos sencillos para obtener el contenido inicial de lactosa de cada una de las muestras, lo que permitió calcular la cantidad de lactosa para estandarizar el suero.

En la fermentación alcohólica ocurren las etapas que se identifican como: pretratamiento de la biomasa, hidrólisis, fermentación alcohólica, separación y purificación del etanol por destilación. Para preparar las muestras de suero estandarizado con lactosa se hizo necesario calcular el balance de masa, la cantidad de suero y lactosa a utilizar.

En la primera fase experimental se utilizaron $10 \mathrm{~L}$ de suero a los cuales se les midió densidad y $\mathrm{pH}$, se calentó hasta la temperatura de 90 a $95^{\circ} \mathrm{C}$, y se ajustó el $\mathrm{pH}$ del suero añadiéndole Ácido Fosfórico $\left(\mathrm{HPO}_{4}\right)$ concentrado hasta $\mathrm{pH}$ 4.5, manteniendo la temperatura con agitación por 30 min con el objetivo de precipitar las proteínas insolubles, así como parte de las solubles, y posteriormente se filtró para eliminar las proteínas. Mediante el Lacto Star se caracterizó el suero desproteinizado y se le midió densidad, Brix, y $\mathrm{pH}$. Con los resultados obtenidos por el Lacto Star respecto a la lactosa presente en el suero se calculó la cantidad de lactosa a añadir para estandarizar el suero y alcanzar las concentraciones de 8 y $10^{\circ}$ Brix. Del volumen total de suero se utilizaron $6 \mathrm{~L}$, los cuales fueron divididos en dos recipientes de $3 \mathrm{~L}$ cada uno, a ambos se les añadió la lactosa para estandarizar según la concentración deseada, $\mathrm{NH}_{3} \mathrm{PO}_{4}$ y levadura, se mantuvo en aireación y agitación por un periodo de 3 horas para garantizar el inicio del proceso de fermentación, concluido ese tiempo se puso a fermentar en condiciones anaerobias, realizándose 3 réplicas de $1 \mathrm{~L}$ por cada concentración.

En las técnicas utilizadas para la hidrólisis de la lactosa se probaron varias técnicas siguiendo el 
procedimiento de hidrólisis ácida. Se pusieron a calentar 2 vasos de precipitado con $200 \mathrm{ml}$ de agua destilada en cada uno de ellos hasta $55^{\circ} \mathrm{C}$, luego se disolvieron en cada uno $25 \mathrm{~g}$ de lactosa. Uno de ellos se puso a enfriar y se le midió $\mathrm{pH}$ y ${ }^{\circ}$ Brix y se tomó como solución patrón. EI otro se mantuvo en calentamiento hasta alcanzar los $85-90^{\circ} \mathrm{C}$, luego se le añadió en caliente $\mathrm{HCl}$ al $1 \mathrm{~N}$ y se dejó a esta temperatura por $50 \mathrm{~min}$, posteriormente se puso a enfriar por diez minutos para parar o apagar la reacción, seguido se neutralizó el ácido con $\mathrm{NaOH}$ al $1 \mathrm{~N}$ ajustando el $\mathrm{pH}$ a valores por encima de 6 . Siguiendo esta misma técnica se realizó otra hidrólisis, pero utilizando $\mathrm{HCl}$ concentrado e $\mathrm{NaOH}$ al $4 \mathrm{~N}$.

\section{Producción de etanol a partir de suero.}

A partir de desechos orgánicos como el suero de leche (fuente de lactosa), con el desarrollo de procesos biotecnológicos se puede producir etanol, utilizando el cultivo de Saccharomyces cerevisiae, es necesario realizar la hidrólisis de la lactosa para obtener glucosa y galactosa como sustratos, reacción mediada por la $\beta$-galactosidasa. Al respecto, Montes de Oca et al.
(2018) refieren que, la lactasa, o también denominada $\beta$ galactosidasa, es una enzima de gran interés biotecnológico por razones nutricionales e industriales, ya que es de utilidad para el desdoblamiento de la lactosa. La enzima causa la hidrólisis del enlace $\beta-1,4$ de la lactosa, y la desdobla en sus azúcares simples, glucosa y galactosa, originando un producto con gran poder edulcorante y con gran aporte a un proceso de fermentación alcohólica.

El método común para hidrolizar la lactosa en la producción de productos lácteos es agregar lactasa. La adición de esta enzima no requiere equipo de procesamiento adicional y la lactasa está ampliamente disponible. El uso de lactasa para hidrolizar la lactosa permite el uso de microorganismos que no producen $\beta$-galactosidasa para la fermentación. Hughes et al. (2018).

\section{Levaduras del género}

\section{Saccharomyces cerevisiae}

Pocas levaduras pueden absorber lactosa para producir etanol. Kluyveromyces marxianus es un microorganismo ampliamente 
utilizado que convierte la lactosa en etanol en la industria. Otros microorganismos utilizados en la producción industrial de alimentos y bebidas se han probado a escala experimental para determinar si son adecuados para convertir lactosa en bioetanol. Estos organismos incluyen Kluyveromyces lactis, Saccharomyces cerevisiae y Escherichia coli (Hughes et al.2018).

Estas levaduras son lo que se denominan: organismos anaeróbicos facultativos, es decir que pueden desarrollar sus funciones biológicas sin oxígeno. Se puede decir que el $96 \%$ de la producción de etanol la llevan a cabo hongos microscópicos, diferentes especies de levaduras, entre las que se encuentran principalmente Saccharomyces cerevisiae. La resistencia de esta levadura a las concentraciones de etanol es de $20 \%$ de concentración en volumen. La composición del mosto es un factor fundamental en la calidad de fermentación por las levaduras, ya que esto juega un papel importante en el desarrollo de la roma y color en la estabilidad del producto final (Luna,2017).
El uso más extendido está enmarcado en la panificación y en las industrias de fabricación de cerveza, vinos y alcohol. La levadura inactivada por temperatura se usa como fuente de nutrimentos en alimentación animal y humana, tanto en forma de levadura íntegra como a partir de sus derivados (Sures et al., 2016).

\section{Preparación de las soluciones.}

Se prepararon soluciones de $\mathrm{HCl}$ al $1 \mathrm{~N}$, para ello se midieron $9.7 \mathrm{ml}$ de $\mathrm{HCl}$ concentrado con una pipeta y se diluyeron en $100 \mathrm{ml}$ de agua destilada. También se preparó $\mathrm{NaOH}$ al 1 y al $4 \mathrm{~N}$. Para preparar el $\mathrm{NaOH}$ al $1 \mathrm{~N}$ se pesaron $4 \mathrm{~g}$ de $\mathrm{NaOH}$ y se diluyeron en $100 \mathrm{ml}$ de agua destilada y para la solución al $4 \mathrm{~N}$ se pesaron $16 \mathrm{~g}$ de $\mathrm{NaOH}$ en $100 \mathrm{ml}$ de agua destilada. Se utilizaron además soluciones al $2 \%$ de glucosa, galactosa y lactosa, además de permanganato de potasio $(\mathrm{KMnO} 4)$ que fueron preparadas por especialistas del departamento de análisis químico para su uso común en diferentes investigaciones. 


\section{Primera fase experimental}

Para alcanzar los ${ }^{\circ}$ Brix deseados en el suero fue necesario calcular mediante balance de masas la cantidad de lactosa para estandarizar, además de las cantidades añadidas al suero estandarizado de $\mathrm{NH} \quad 3 \mathrm{PO} 4$ y levadura para lafermentación.

Según los resultados obtenidos en las corridas experimentales con el suero lácteo suplementado y bajo régimen se pudo afirmar que la levadura utilizada no fue capaz de iniciar el proceso fermentativo, esta se inmovilizó en el fondo del reactor sin observarse aumento en su crecimiento. Quedando descartado que la levadura utilizada por la Industria Alcoholera cubana en la fabricación de cerveza degrada lactosa, es decir es lactosanegativa.

La primera fase experimental condujo la investigación a la necesidad de lograr en el suero lácteo un sustrato más efectivo para la fermentación y realizar las pruebas con la otra opción de levadura Saccharomyces cerevisiae que se fabrica en el país para la obtención devinos.
Hidrólisis de la lactosa: La hidrólisis de la lactosa se realizó siguiendo el procedimiento de hidrolisis ácida, los resultados obtenidos para ambos métodos fueron los siguientes:

Hidrólisis con $\mathrm{HCl}$ al $1 \mathrm{~N}$ : Las pruebas realizadas no mostraron diferencias entre las muestras analizadas de soluciones de lactosa.

Hidrólisis con $\mathrm{HCl}$ concentrado: Se obtuvieron resultados positivos para este método, pues con las pruebas realizadas a las muestras se evidenció diferencias entre las muestras hidrolizadas y $\sin$ hidrolizar.

Para comprobar la hidrólisis de la lactosa se desarrolló la Prueba de Polarimetría con los siguientes resultados: Con una solución de $100 \mathrm{ml}$ de lactosa, sin hidrolizar, la polaridad fue de 10,2; hidrolizada con $\mathrm{HCl}$ al $1 \mathrm{~N}$, la polaridad fue 10,2, hidrolizada con $\mathrm{HCl}$ concentrado, la polaridad de 12,7

Según los resultados obtenidos se pudo asumir que hubo hidrólisis utilizando $\mathrm{HCl}$ concentrado, se aprecia diferencia entre los valores de las polaridades, lo cual es una 
propiedad intrínseca de cada sustancia y muestra la variación de la lectura del polarímetro de la muestra hidrolizada con $\mathrm{HCl}$ concentrado, aunque la polaridad no se utiliza para identificar sustancias, cuando se aprecian diferencias se puede afirmar que ha ocurrido una transformación por el proceso de hidrólisis.

Cromatografía de capa fina: Mediante esta prueba quedó demostrado que ocurrió un desdoblamiento de la lactosa en glucosa y galactosa y aunque la hidrólisis no fue total, si ocurrió el proceso de hidrólisis. A pesar de observarse que las manchas en la placa en algunas zonas no se delimitaron como lo esperado, se pudo apreciar la diferencia entre la muestra de lactosa hidrolizada en comparación con las muestras de glucosa, galactosa y lactosa sin hidrolizar por el desplazamiento de las muestras de derecha a izquierda.

Colorimetría: Los resultados obtenidos permiten afirmar que ocurrió el proceso de hidrólisis, pues en la muestra con lactosa hidrolizada se evidenció el cambio de coloración a carmelita, lo cual no ocurrió en los tubos que contenían soluciones de glucosa, galactosa y lactosa sinhidrolizar.

\section{Resultados}

Para calcular la cantidad de lactosa a añadir al suero se partió de su contenido en el suero desproteinizado y se hicieron cuatro mediciones los días 7, 8, 16 y 17 de mayo, en los mismos la densidad del suero inicial, fue de 1,032 g/l, 1,032 $\mathrm{g} / \mathrm{l}, \quad 1,029 \quad \mathrm{~g} / \mathrm{l} \quad$ y $\quad 1,027 \quad \mathrm{~g} / \mathrm{l}$, respectivamente. La concentración deseada de latosa fue de $125 \mathrm{~g} / \mathrm{l}$, $100 \mathrm{~g} / \mathrm{l}, 75 \mathrm{~g} / \mathrm{l}$ y $50 \mathrm{~g} / \mathrm{l}$, en las mencionadasmediciones.

Los valores del cálculo de la cantidad de lactosa para estandarizar el suero mediante el balance de masa, considerándole a la lactosa con un $4 \%$ de humedad en las diferentes lecturas con una concentración de lactosa deseada de $125 \mathrm{~g} / \mathrm{l}, 100 \mathrm{~g} / \mathrm{l}$, $75 \mathrm{~g} / \mathrm{l}$ y $50 \mathrm{~g} / \mathrm{l}$ respectivamente y la lactosa calculada para estandarizar el suero fue en cada caso de 66,69 g, 45,38 g, 32,17 g y 13,45 g. El porciento de sólidos totalesobtenido fue $15,0-13,0-10,0 \quad$ - 8,0, correspondientemente. 


\section{Procedimiento utilizado a partir de lactosa hidrolizada}

El procedimiento utilizado con lactosa hidrolizada para la fermentación alcohólica del lactosuero reflejó resultados positivos, observándose la actividad de la levadura además del crecimiento de su masa. Al utilizar para el proceso fermentativo como sustrato lactosuero estandarizado a diferentes concentraciones de lactosa hidrolizada se pudo apreciar el desarrollo de la fermentación, lo cual no ocurrió en la primera fase experimental, quedando demostrado que la levadura Saccharomyces cerevisiae utilizada por la Industria Alcoholera cubana en la fabricación de vinos es activa en el lactosuero con la lactosa hidrolizada a pesar del resto de los componentes que quedan presentes en el suero después de los tratamientos de descreme y desproteinización descritos en el trabajo.

Los resultados obtenidos muestran que durante el proceso fermentativo la levadura consumió nutrientes, aumentando su actividad en las muestras en las que las concentraciones de lactosa eran más elevadas, específicamente en la concentración de $125 \mathrm{~g} / \mathrm{l}$ en la que se observa que la variación de los Brix fue mayor con respecto a las demás concentraciones estudiadas, es decir que mientras aumentaban las concentraciones de lactosa el consumo de sustrato por la levadura se incrementó.

\section{Determinaciones realizadas al etanol obtenido de la destilación.}

Los resultados obtenidos de las determinaciones indican que a partir de las concentraciones de lactosa de $75 \mathrm{~g} / \mathrm{l}$ hasta $125 \mathrm{~g} / \mathrm{l}$ aumentó la cantidad de etanol obtenido de la destilación, destacándose la concentración de $125 \mathrm{~g} / \mathrm{l}$ de lactosa, en la cual se obtuvo el mayor grado alcohólico, siendo este el mejor resultado que se llegó a alcanzar en una de las réplicas (6ªlcohólico), encontrándose este valor por encima de lo pronosticado. Los resultados indican aumento del etanol obtenido con el incremento del contenido de lactosa hidrolizada en el suero. Con la concentración de lactosa de $50 \mathrm{~g} / \mathrm{l}$ el etanol obtenido resultó inferior a lo esperado, pues al ser comparados con investigaciones realizadas por Padín y Díaz, (2006), donde se hace alusión a procesar suero sin concentrar, con $4.5 \%$ de lactosa, se 
obtuvo etanol potable por destilación (2.8\%), utilizando cepas de Kluyveromyces fragilis, K. Lactis y Candidakefir (pseudotropicalis). A partir de las concentraciones de $75 \mathrm{~g} / \mathrm{L}$ de lactosa hidrolizada los resultados aumentaron y están en correspondencia con los referenciados por Padín y Díaz, (2006), donde se realizaron estudios con las cepas citadas anteriormente y concentraciones de lactosa de $130 \mathrm{~g} / \mathrm{l}$.

El etanol teórico, el tiempo de fermentación y el etanol final aumentaron con el incremento de las concentraciones de lactosa hidrolizada. Los resultados a partir de las concentraciones de 75 hasta $125 \mathrm{~g} / \mathrm{l}$ de lactosa están en correspondencia con los obtenidos en otros estudios realizados utilizando la levadura Kluyveromyces fragilis la cual produjo más etanol mientras mayor fue la concentración de lactosa en el substrato. Además, al ser mayor la concentración de lactosa en el medio de fermentación, más tarde apareció la máxima concentración de etanol en el mismo Padín y Díaz, (2006). A concentraciones de $50 \mathrm{~g} / \mathrm{l}$ de lactosa la levadura continuó produciendo etanol hasta el final del experimento (40 horas) como era de esperar, debido a la presencia de glucosa en el sustrato, pero la cantidad producido no es significativa y la evidencia de la fermentación la hizo despreciable. Los rendimientos y la productividad también crecen, pero resultan positivos a partir de concentraciones de lactosa de75g/l.

\section{Procesamiento estadístico}

Mediante el diseño de Factor categórico simple se procesaron los resultados de las corridas y se aprecian en la Tabla 1.

Tabla 1. Corridas procesadas según la concentración de lactosa.

\begin{tabular}{|c|c|c|c|c|c|}
\hline Run & $\begin{array}{c}\text { Conc. Lactosa } \\
(\mathbf{g} / \mathbf{m l})\end{array}$ & Var. ${ }^{\circ}$ Brix & Alcohol (g/L) & Tiempo(h) & $\begin{array}{c}\text { \%Etanol } \\
(\% \mathbf{v}-\mathbf{v})\end{array}$ \\
\hline 1 & 50.0 & 1.0 & 8.0 & 40.0 & 0.8 \\
\hline 2 & 50.0 & 1.0 & 8.0 & 40.0 & 0.8 \\
\hline 3 & 75.0 & 3.0 & 27.6 & 48.0 & 2.8 \\
\hline 4 & 75.0 & 3.0 & 27.6 & 48.0 & 2.8 \\
\hline 5 & 100.0 & 6.0 & 39.3 & 54.0 & 4.0 \\
\hline
\end{tabular}




\begin{tabular}{|c|c|c|c|c|c|}
\hline 6 & 100.0 & 6.3 & 39.3 & 54.0 & 4.0 \\
\hline 7 & 125.0 & 9.0 & 58.6 & 63.0 & 6.0 \\
\hline 8 & 125.0 & 8.5 & 49.5 & 61.0 & 5.0 \\
\hline
\end{tabular}

El procesamiento de los valores de las corridas que se exponen en la Tabla 1 mediante las pruebas de Anova y Duncan exponen los siguientes resultados: La prueba Anova en relación con la concentración de lactosa y el alcohol obtenido, permitió determinar que existían diferencias significativas entre las muestras de diferentes concentraciones de lactosa. Para analizar entre cuales concentraciones de lactosa existía mayor diferencia se realizó una prueba Duncan y los resultados se muestran a continuación.

En las cuatro concentraciones de lactosa utilizada para la fermentación alcohólica se observó que aumentó desde las concentraciones de $50 \mathrm{~g} / \mathrm{l}$ hasta $125 \mathrm{~g} / \mathrm{l}$, pero se destaca que una diferencia significativa entre las pruebas de $50 \mathrm{~g} / \mathrm{l}$ y $75 \mathrm{~g} / \mathrm{l}$ de lactosa es mayor que la existente entre una y otra prueba sucesiva. Los resultados obtenidos de la prueba Anova para la variación de los Brix con respecto a la concentración de lactosa, en el cual se observa que existen disparidades entre las muestras de concentraciones de lactosa diferentes.

En la prueba Duncan para variación de oBrix y concentración de lactosa los resultados obtenidos reflejan diferencias significativas entre los @Brix para cada corrida estudiada, observando más acentuado entre 75 g/l y $100 \mathrm{~g} / \mathrm{l}$ de lactosa, lo que puede explicarse por la composición de sólidos disueltos en el suero de otros componentes como sales minerales.

El procesamiento de los resultados estadísticos para la concentración de lactosa con relación al tiempo de fermentación que se obtuvieron a través de la prueba Anova reflejaron diferencias, además se realizó una prueba Duncan para Tiempo y concentración de lactosa.

Los resultados obtenidos demostraron que hay disparidad del tiempo de fermentación entre las corridas realizadas, y a medida que aumentó la concentración de lactosa 
aumentó el tiempo de fermentación. La prueba Anova para la relación entre la concentración de lactosa y el por ciento de etanol, demostró que existían diferencias entre el por ciento de etanol obtenido para cada concentración de lactosa.

Los resultados obtenidos mediante la prueba Duncan para concentración de lactosa y por ciento de etanol muestran que hay desigualdades entre las concentraciones estudiadas, destacándose la mayor diferencia significativa entre las corridas de 50 g/l y 75 g/l, apreciándose que a medida que aumenta la concentración de lactosa se incrementan los por cientos de etanol obtenidos. Con los resultados obtenidos en la Anova de correlación se pudo determinar que existía correlación entre las concentraciones de lactosa, el tiempo de fermentación y los gramos de etanol obtenidos lo cual se muestran en los valores de correlación de 98.93 y 97.1 respectivamente y se aprecia que a medida que aumentaba la concentración de lactosa se obtuvieron mayores valores de las variables respuestas estudiadas como se muestra en el gráfico 1.

Figura 1. Resultados de la Fermentación.

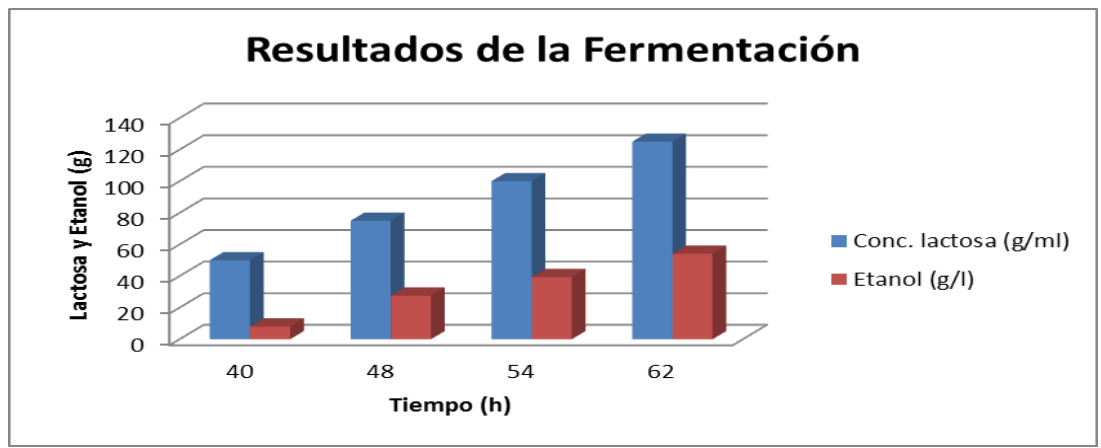

Se obtuvieron las siguientes ecuaciones de correlación:

Alcohol $=-74.4912+2.09272$ * Tiempo.
Alcohol $=-20.21+0.5994$ * Conc_lactosa

Según los resultados que se muestran en el gráfico: 1 se observó un comportamiento creciente, es decir la concentración de lactosa, el tiempo y los gramos de etanol obtenidos presentaron una estrecha 
relación, ya que a medida que se aumentaba la concentración de lactosa, el tiempo de fermentación se hacía cada vez mayor, incrementándose de esta manera la cantidad de etanol obtenido con respecto a cada concentración.

\section{Conclusiones}

Las determinaciones realizadas permitieron la caracterización del suero, mostrando para la lactosa resultados inferiores a los registrados en la literatura analizada, la levadura Saccharomyces cerevisiae utilizada en la Industria Alcoholera Cubana no fermenta lactosa como sustrato en el proceso fermentativo.

Los resultados de hidrólisis parcial de la lactosa permitieron obtener en el proceso fermentativo ácido porcientos en volúmenes de etanol por encima de los propuestos a partir de concentraciones de lactosa de 75 $\mathrm{g} / \mathrm{l}$, el incremento de las concentraciones de lactosa hasta 125 g/l está en correspondencia con los mejores rendimientos y productividad del proceso fermentativo.

\section{Bibliografía}

Aráuz, M. (2020). Fermentación de lactosuero para la obtención de etanol y su uso en cervezas y bebidas saborizadas Revisión de Literatura, Escuela Agrícola Panamericana, Zamorano, Honduras, noviembre. Disponible en: https://bdigital.zamorano.edu/ handle/11036/6919.

Espinoza, H., Mendieta, E. (2018). Efectos de la fermentación láctica del lactosuero y alcohólica del mucílago de cacao en la concentración final de una bebida alcohólica. (Tesis de grado). Escuela Superior Politécnica Agropecuaria de Manabí Manuel Félix López. Bolívar, Manabí, Ecuador. Disponible en: http://repositorio.espam.edu.e c/handle/42000/891

Hughes, P., Risner, D., Goddik, L. (2018). Suero a Vodka. En suero: propiedades biológicas y usos alternativos. London. Disponible en: https://www.intechopen.com/ books/whey-biologicalproperties-and-alternativeuses/whey-to-vodka

Jácquez-Velázquez, L., Soto-Cruz, N., Rutiaga-Quiñones, O., Páez-Lerma, J. (2015). Evaluación de las variables de fermentación para la 
producción de etanol a partir de lactosuero. XVI Congreso Nacional de Biotecnología y Bioingeniería. 21 al 26 de junio 2015. Guadalajara México.

Lawton, M., Alcaine, S. (2019). Leveraging endogenous barley enzymes to turn lactose- containing dairy byproducts into fermentable adjuncts for Saccharomyces cerevisiae-based ethanol fermentations. Journal of dairy science. 102(3), 20442050.Disponible en: https://doi.org/10.3168/jds.20 18-15586

Londoño, M., Sepúlveda, J., Hernández, A., Parra, J. (2008). Bebida fermentada de suero de queso fresco inoculada con Lactobacillus casei. Revista Facultad Nacional de Agronomía. Medellín. 2008,61(1), 44094421 [fecha de Consulta 7 de octubre de 2020]. ISSN:03042847. Disponible en: https://www.redalyc.org/articu lo.oa?id=179914077017

Luna, L. (2017). Manual de control de calidad de la Cervecería Gourmet del Golfo SA de CV. Universidad Tecnológica del Centro de Veracruz. México. 42p. Disponible en: http://reini.utcv.edu.mx/handl e/123456789/294

Montesdeoca, R., Intriago, R., Vera, P., Benítez, I. (2018). Efecto de la adición de lactasa y sacarosa en una bebida isotónica utilizando lactosuero. Revista chilena de nutrición. 45(4),316-322.

Padín, C., Díaz, M. (2009). Fermentación alcohólica del lactosuero por Kluyveromyces marxianus y solventes orgánicos como extractantes. Revista de la Sociedad Venezolana de Microbiología. 29(2), 110-116.

Panesar, P., Kennedy, J., Knill, C., Kosseva, M. (2010). Production of $L(+)$ Lactic Acid using Lactobacillus casei from Whey. Brazilian archives of biology and technology. Vol. 53.p219-226.

Parra, R. (2009). Lactosuero: importancia en la industria de alimentos. Revista Facultad Nacional de Agronomía. Medellín. Vol. 62. 0304- 2847.

Rodríguez, D., Aldo, H. (2017). Desarrollo de una bebida fermentada de suero con la adición de jugo de Aloe vera y pulpa de fruta. Tecnologica Quimica, 37(1), 2-3, abril de 2017

Suáres, C., Garrido, N., Guevara, C. (2016).

Levadura Saccharomyces Cerevisiae y la producción de alcohol. ICIDCA (Instituto Cubano de Investigaciones de los Derivados de la Caña de Azúcar), 21 -23. 
Trevan MD. (1990) Biotecnología:

Principios biológicos.

Zaragoza: Acribia; xiii, 284.

ISBN: 84-200-0671-8.

Vargas, X. (2017). Evaluación de la producción de etanol a partir de lactosuero a nivel de biorreactor (bioflo 110) utilizando Kluyveromyces marxianus y Kluyveromyces lactis como agentes fermentativos. (Tesis de grado). Universidad de la Salle Facultad de Ingeniería. Bogotá, Colombia. 